\title{
Dynamic construction of virtual manufacturing cell considering workload balancing and machine failure
}

\author{
Zhe Lv ${ }^{*}$, Junjie Fan, and Lipeng Wang \\ Jiangsu University of Science and Technology, Zhenjiang, Jiangsu212003, China
}

\begin{abstract}
Keywords: virtual manufacturing cell, machine failure, dynamic construction, PSO-GA hybrid algorithm.

Abstract. Machine is the key element in manufacturing system. Machine failure will lead to order delivery delay and production cost rise. Aiming at the random disturbance of equipment failure, a dynamic non-linear programming model of virtual manufacturing cell (VMC) is established, which aims to minimize the equipment cost, logistics transportation cost, delay delivery cost and the differences of workload among cells, based on the comprehensive consideration of equipment reliability, process route constraints, variable processing path and workload. Due to the high complexity of the problem, the hybrid particle swarm optimization algorithm is used to solve the model, and the rationality and effectiveness of the method are verified by a numerical example. Experimental results show that this method has advantages in reducing the cost of cell reconfiguration, improving the utilization of equipment and shortening the completion time.
\end{abstract}

\section{Introduction}

Virtual manufacturing cell is a new development of cell production mode and an advanced form of the production organization. The virtual manufacturing cell would not rearrange the physical location of the devices, and logically clusters the resources to form cells, which greatly reduces the cost of moving the device and the adjustment time [1]. VMC combines the advantages of cell-layout and technological-layout, which can fully exploit the ability of production resources in manufacturing system, and can well adapt to the current requirements of multi variety and small batch production [2].

In the process of virtual manufacturing cell production, with the machine failure occurs, the original virtual, manufacturing cell structure may not meet the needs of later processing, so it is necessary to conduct virtual cell reconfiguration. Many researches have been done on the problem of virtual manufacturing cell and dynamic construction.

In regard to the virtual manufacturing cell, Liang and fung [3] introduced a collaborative mechanism to support real-time scheduling of virtual manufacturing cell.

\footnotetext{
${ }^{*}$ Corresponding author. Email: polylv@,foxmail.com
} 
Hamedi et al.[4] constructed a capability-based multi-objective model of virtual manufacturing cell, in which workers differ in skill level and heterogeneity. Kesen et al.[5] respectively explained the three types of production organization methods: virtual unit, traditional unit and process-based production, and verified that the virtual unit production organization method is superior to the other two production organization methods in terms of performance through the method of system simulation. Nomden and Zee [6] combined the production organization of the parallel machining workshop with the production organization of the virtual manufacturing cell. The results of the study show that a moderate investment in production planning, equipment and reusable resources will help improve the production capacity of the workshop.

Celluar dynamic construction has attracted many interests, Mehrabad [7] build a biobjective possibilistic programming model for the dynamic formation of virtual manufacturing cells integrated in procurement, production and distribution planning. Mahdavi et al.[8] build a virtual manufacturing cell based on fuzzy goal planning, so that the machine-family, part-family, and worker-family in the cell can meet the dynamic changes of market demand. Rezazadeh et al.[9] constructed two integrated virtual manufacturing cell models in consideration of factors such as production planning, dynamic reconstruction, and worker needs. After linearizing the models, they were solved by the particle swarm optimization algorithm.

The existing literature on virtual manufacturing unit and unit reconfiguration rarely considers the situation of cross-unit transportation, but in actual production, the phenomenon of cross-unit transportation often occurs and will have a great impact on the production logistics system. In addition, there are differences in workload between different units. If you do not pay attention to maintaining the relative balance of the workload between the units during the reconstruction of the virtual manufacturing unit, it will lead to problems such as an increase in the idle rate of processing equipment and a serious backlog of work-in-process inventory. Appeared.

The fault-type disturbance represented by the machine fault is a common disturbance event in the production system [10]. The occurrence of machine failure may cause the production resource allocation method of the initial virtual manufacturing unit to fail to meet the production requirements, and the original virtual manufacturing unit's organizational structure needs to be reconstructed. Therefore, in view of equipment fault disturbance, this paper constructs a multi-objective nonlinear integer programming model with the lowest equipment usage cost, logistics transportation cost, and tardiness delivery cost, and the smallest equipment load difference between units, and uses the PSO-GA hybrid algorithm to compare the model. Solve. A calculation example verifies the rationality of the proposed model and the effectiveness of the algorithm.

\section{Modeling construction and algorithm design}

\subsection{Problem description}

The occurrence of equipment failure is usually unpredictable and random. In order to maintain the stability of the production system, the affected ordinary new orders need to be inserted into the current virtual cell for processing. Therefore, enterprises need to realize the rapid reconfiguration of virtual cell based on the existing virtual cell construction scheme.

The dynamic construction problem of virtual cell under the disturbance of equipment failure can be described as follows: in the workshop, there are $c$ original virtual cells, $m$ machines that available for processing, $i$ workpieces and each workpiece contains $j$ operations. When the machine failure occurs and the processing capacity of the remaining 
equipments are insufficient to meet the demand of production, the virtual cell needs to be reconstructed to ensure the efficient and stable production.

In order to better build the model, the following assumptions are made for the virtual cell manufacturing system: (1) The upper limit of the number of virtual units is known, and the upper and lower limits of the number of machine tools in each virtual unit are determined in advance; (2) The demand of each stage of each workpiece is known; (3) each workpiece needs different types of machine tools to process multiple processes, and the process sequence and processing time are known; (4) each machine tool can only process one process at the same time; (5) the same process of the same workpiece can only be processed on one machine; (6) when the equipment failure occurs, if the existing equipment has residual processing energy, the machine tool can only process one process If the force can not meet the production demand, the virtual cell reconstruction is only carried out for the process of the unprocessed workpiece, that is, the process of the workpiece being processed on a machine tool is not affected until the processing is completed; the process of the finished workpiece is not affected, and the reconstruction is not considered.

\subsection{Dynamic construction model of virtual manufacturing cell}

(1) Input parameters

$c$ - Index for virtual manufacturing cells $(c=1,2, \ldots, C)$

$\bar{c}$ — Index for reconstructed virtual manufacturing cells $(\bar{c}=1,2, \ldots, \bar{C})$

$h-$ Index for time periods $(h=1,2, \ldots, H)$

$p$-Index for parts $(p=1,2, \ldots, P)$

$j_{p}$-Index for operations belonging to part $p\left(j_{p}=1,2, \ldots, J_{p}\right)$

$m$-Index for machines $(m=1,2, \ldots ., M)$

$D_{p h} \longrightarrow$ Demand for the part $p$ in period $h$

$\gamma_{j_{p}}^{\text {inter }}$ Inter-cell movement cost for per $j_{p}$

$\gamma_{j_{p}}^{\text {inter }}$ Intra-cell movement cost for per $j_{p}$

$D L_{p h} \longrightarrow$ Delivery time of part $p$ in period $h$

$\Gamma_{p} \longrightarrow$ Unit delay penalty cost for per $p$

$B_{p} \longrightarrow$ The batch size for $p$

$t_{j_{p} m} \longrightarrow$ Processing time required to perform $j_{p}$ on machine $m$

$a_{m}-$ Cost of machine $m$

(2) Decision variables

$\alpha_{p h}$ - If the part $p$ is planned to produce in period $h, \alpha_{p h}=1$; otherwise, the value is 0

$\beta_{j_{p} m}$-If the operation $j_{p}$ can be produced on machine $m, \beta_{j_{p} m}=1$;otherwise, the value is 0

$\pi_{p c h}$ If at least one operation of part $p$ is planned in cell $c$ in period $h, \pi_{p c h}=1$; otherwise, the value is 0 
$\vartheta_{j_{p} m c h}$ - If operation $j_{p}$ is planned on machine $m$ in cell $c$ in period $h, \vartheta_{j_{p} m c h}=1$; otherwise, the value is 0

$F T_{p h}-$ Completion time of part $p$ in period $h$

$S T_{p} \longrightarrow$ Processing start time of part $p$

$E T_{p} \longrightarrow$ Processing end time of part $p$

$D T_{p h} \longrightarrow$ Delivery delay time of part $p$ in period $h$

$O U_{h}-$ Utilization rate of all machines in period $h$

$C U_{c h}$ Utilization rate of machines in cell $c$ in period $h$

$N_{m c h} \longrightarrow$ Number of machines in cell $c$ in period $h$

$T_{m h}$ Time processing capacity of machine $m$ in period $h$

$J_{c m}-$ Number of operations processed on the equipment at a certain time in cell $c$

(3) Model construction

(1) Production cost

Whenever the fault disturbance occurs, the dynamic construction of the original virtual manufacturing cell will lead to the increase of production cost:

(a) Operating cost of processing equipments

$$
f_{1}=\sum_{h=1}^{H} \sum_{c=1}^{C} \sum_{p=1}^{P} \sum_{j_{p}=1}^{J_{p}} \sum_{m=1}^{M} a_{m} D_{p h} t_{j_{p} m} \vartheta_{j_{p} m c h}
$$

(b) Logistics transportation cost

Due to the fact that virtual manufacturing cell (VMC) does not change the physical layout of equipment, but logically forms interrelated virtual dynamic entities for workpiece production, the change of production plan will inevitably lead to the frequent flow of production tasks among manufacturing equipment, resulting in the disorder of logistics and the substantial increase of logistics cost. Therefore, it is necessary to consider the logistics cost index in cell reconfiguration.

In the case of cell-inter transportation, the logistics cost could be expressed as follows:

$$
\begin{array}{r}
C_{\text {inter }}=\sum_{h=1}^{H} \sum_{p=1}^{P} \gamma_{j_{p}}^{\text {inter }}\left\lceil\frac{D_{p h}}{B_{p}}\right\rceil \sum_{j=1}^{(J-1)_{p}} \sum_{c=1}^{C} \times \\
\left|\sum_{m=1}^{M} \vartheta_{(j+1)_{p} m c h}-\sum_{m=1}^{M} \vartheta_{(j+1)_{p} m c h}\right|
\end{array}
$$

In the case of cell-intra transportation, the logistics cost is expressed as follows: 


$$
\begin{aligned}
C_{\text {intra }}= & \left.\sum_{h=1}^{H} \sum_{p=1}^{P} \gamma_{j_{p}}^{\text {intra }} \mid \frac{D_{p h}}{B_{p}}\right\rceil \sum_{j=1}^{(J-1)_{p}} \sum_{c=1}^{C}\left(\sum_{m=1}^{M}\left|\vartheta_{(j+1)_{p} m c h}-\vartheta_{j_{p} m c h}\right|\right. \\
& \left.-\left|\sum_{m=1}^{M} \vartheta_{(j+1)_{p} m c h}-\sum_{m=1}^{M} \vartheta_{j_{p} m c h}\right|\right)
\end{aligned}
$$

Where, denotes the largest integer less than the internal value.

The total cost of logistics transportation can be expressed as:

$$
f_{2}=\rho \cdot C_{\text {inter }}+\sigma \cdot C_{\text {intra }}
$$

In Equation(4), $\rho$ and $\sigma$ are weight coefficients.

(c) Penalty cost of order delay delivery

$$
f_{3}=\sum_{h=1}^{H} \sum_{p=1}^{P} D_{p h} \Gamma_{p} D T_{p h}
$$

Therefore, under the condition of equipment failure, the production cost of virtual manufacturing cell is expressed as follows:

$$
F_{1}=\left\{f_{1}, f_{2}, f_{3}\right\}
$$

(2) Workload balance

In this section, the utilization rate of processing equipment in different cells is used as an index to measure the workload difference between cells. The optimization of this index will be conductive to maintain the relative balance of workload among cells, so as to ensure the orderly and efficient production. The expression is as follows:

$$
F_{2}=\sum_{h=1}^{H} \sum_{c=1}^{C}\left|C U_{c h}-O U_{c h}\right|
$$

The dynamic multi-objective programming model of virtual manufacturing cell is shown as follows:

$$
F=\left\{F_{1}, F_{2}\right\}
$$

S.t.

$$
\begin{aligned}
& \sum_{c=1}^{C} \sum_{m=1}^{M} \beta_{j_{p} m} \vartheta_{j_{p} m c h}=D T_{p h} \quad \forall j, p, h \\
& J_{c m}=\{0,1\} \quad \forall c, m \\
& \vartheta_{j_{p} m c h} \leq \beta_{j_{p} m} \quad \forall j, p, h, c, m
\end{aligned}
$$




$$
\begin{aligned}
& \left(F T_{p h}-D L_{p h}\right) \leq D T_{p h} \quad \forall p, h \\
& 0 \leq D T_{p h} \quad \forall p, h \\
& E T_{p} \leq S T_{(p+1)} \\
& C U_{c h}=\frac{\sum_{m=1}^{M} \sum_{p=1}^{P} \sum_{j_{p}=1}^{J_{p}} D_{p h} \beta_{j_{p} m} \vartheta_{j_{p} m c h}}{\sum_{m=1}^{M} N_{m c h} T_{m h}} \forall c, h \\
& O U_{h}=\frac{\sum_{c=1}^{C} \sum_{m=1}^{M} \sum_{p=1}^{P} \sum_{j_{p}=1}^{J_{p}} D_{p h} \beta_{j_{p} m} \vartheta_{j_{p} m c h}}{\sum_{c=1}^{C} \sum_{m=1}^{M} N_{m c h} T_{m h}} \quad \forall h \\
& C U_{c h}, O U_{h}, N_{m c h}, F T_{p h} \geq 0 \text { and integer }
\end{aligned}
$$

In the aboved model, Equation (9) ensures that the same process of the workpiece in the same cycle can only be processed on one equipment; Equation (10) indicates that each equipment can only belong to one virtual unit at a certain time and can only be allocated to one process at most; Equation (11) ensures that all processes can be processed; Equation (12) and Equation (13) are used to calculate the order delivery delay time; Equation (14) ensures that all processes can be processed It means that only when the previous process is completed can the next process be started; Equation (15) is used to determine the utilization rate of machines and equipment in each unit in each production planning period; Equation (16) is used to determine the utilization rate of all machines in each production planning period; Equation (17) is a non negative integer constraint on the decision variables.

\section{Algorithm design}

In this paper, PSO-GA hybrid algorithm is used to solve the multi-objective programming model of the proposed virtual manufacturing cell. According to the characteristics of the research problem, we make changes to the coding structure of the hybrid algorithm and improve the updating method of particles.

\subsection{Particle coding}

We assume that the total number of operations in the cell is $\Phi=\sum_{j=1}^{J} I_{j}$ and a $2 \times \Phi$ dimensional matrix is used to represent and record all the information of particles.

The first line of the matrix is the coding based on the sequence of operations, each element represents the part number, and the n-th occurrence of the part number in sequence represents the $n$-th operation of the part; the second line is the coding based on the 
equipment, each element is the equipment number, which represents the processing equipment selected for each operation. As shown in Figure 1, the code of the fourth column of the particle is, indicating that the processing of the second operation of the first workpiece is assigned to the equipment 3 .

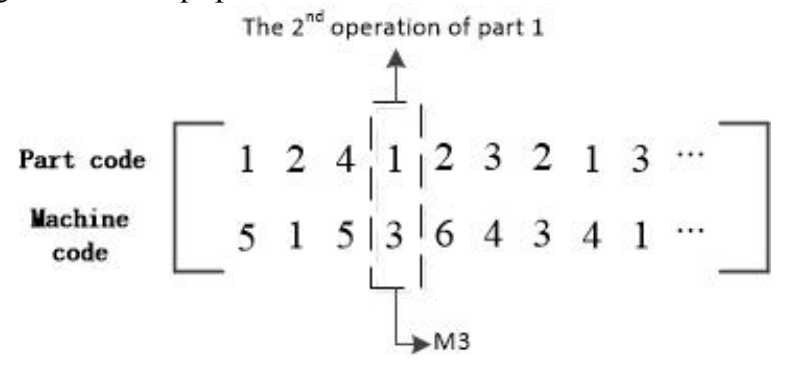

Fig. 1. Particle encoding diagram.

\subsection{Particle updating}

We introduce the crossover operation of genetic algorithm is into particle swarm optimization algorithm. The particle will update the speed and position by imitating the crossover method of chromosome. Specifically, the velocity of the particle is improved by crossing with the current individual optimal solution and the group optimal solution respectively, and the position update of the particle is obtained by crossing the current position and the current velocity of the particle. The velocity position update formula of particles is expressed as follows:

$$
\begin{aligned}
& v_{i}(k+1)=v_{i}(k) \otimes p_{\text {ibest }} \otimes p_{\text {gbest }} \\
& x_{i}(k+1)=v_{i}(k+1) \otimes x_{i}(n)
\end{aligned}
$$

Among them, $k$ represents the number of iterations, $v_{i}$ represents the particle speed, $x_{i}$ represents the particle position, $p_{i b e s t}$ represents the current individual optimal position, $p_{\text {gbest }}$ represents the current population optimal position.

After several iterations, the particle may fall into the local optimal trap. At this time, in order to jump out of the trap, we need to expand the neighborhood search range. We can use particle mutation to update the particle state information and continue to move and search in other areas of the solution space. Specifically, it is a random mutation process for the coding of individual extremum of particles. The formula is as follows:

$$
p_{\text {ibest }}(n+1)=\operatorname{mutation}\left(p_{\text {ibest }}(n)\right)
$$

\section{Numerical experiment}

\subsection{Basic data}

Based on the case data of enterprises, we study the dynamic construction of virtual manufacturing cell in $4 \times 8 \times 8 \times 10 \times 2\left(J_{p} \times P \times M \times C \times H\right)$ environment. The available processing equipment corresponding to each process, the unit cost of logistics 
transportation within / between units, the logistics transportation distance between each equipment and the delivery date of different batches of workpieces are known.

\subsection{Data analysis}

In this paper, the hybrid algorithm of virtual manufacturing cell is realized by MATLAB 2014a. The initial virtual anufacturing cell is constructed according to the production tasks and processing information. The parameters of the algorithm are set as follows: the number of population is 70 , the number of iterations is 120 , the crossover probability is 0.7 , and the mutation probability is 0.3 . Different weights are given to each index in the production cost as: $f_{1} \leftarrow 0.25, f_{2} \leftarrow 0.4, f_{3} \leftarrow 0.35$. Also, the weight coefficients $\rho$ and $\sigma$ are both assigned as 0.5 .

According to the above production information, the initial virtual manufacturing cell is constructed.

It can be found from table 3.4 that three virtual cells are generated respectively for production task and process information planning period 1 and planning period 2, and the three virtual cells are shared by equipment, which effectively reduces the number of task cross cell processing, so as to ensure that the task can be completed in the corresponding virtual cell as far as possible.

Whenever $T=120$, the machine $M_{4}$ breaks down.In order to maintain the production sequence, we could use the method proposed in this paper to reconstruct the initial virtual manufacturing cell, the results juet shown in Table 1 .

Table 1. Initial virtual manufacturing cell scheme.

\begin{tabular}{ccc}
\hline Period & Processing task family & Virtual manufacturing cells \\
\hline \multirow{2}{*}{ Period1 } & P1,P2 & M2,M3,M7 \\
& P4,P5,P6 & M1,M3,M6,M7,M8 \\
& P3,P7 & M2,M3,M8 \\
Period2 & P3,P7 & M2,M3,M8 \\
& P4,P5,P6P,P8 & M1,M3,M5,M7,M8 \\
& P1,P2 & M2,M3,M7 \\
\hline
\end{tabular}

According to the number of cells, logistics cost, equipment utilization, stability index and maximum completion time, the reconfiguration scheme of virtual manufacturing cell proposed in this paper is compared with the scheme of maintaining the original virtual manufacturing cell architecture. The results are shown in Table 2.

Table 2. Reconfiguration of virtual manufacturing cell

\begin{tabular}{cc}
\hline Processing task family & Virtual manufacturing cells \\
\hline $\mathrm{P}_{3}, \mathrm{P}_{7}$ & $\mathrm{M}_{2}, \mathrm{M}_{3}, \mathrm{M}_{8}$ \\
$\mathrm{P}_{4}, \mathrm{P}_{5}, \mathrm{P}_{6}, \mathrm{P}_{8}$ & $\mathrm{M}_{1}, \mathrm{M}_{3}, \mathrm{M}_{5}, \mathrm{M}_{7}, \mathrm{M}_{8}$ \\
$\mathrm{P}_{1}, \mathrm{P}_{2}, \mathrm{P}_{9}$ & $\mathrm{M}_{2}, \mathrm{M}_{3}, \mathrm{M}_{6}$ \\
\hline
\end{tabular}

According to the number of cells, logistics cost, equipment utilization, stability index and maximum completion time, the reconfiguration scheme of virtual manufacturing cell proposed in this paper is compared with the scheme of maintaining the original virtual manufacturing cell architecture. The results are shown in Table 3.

Compared with the inherited virtual cell reconfiguration scheme proposed in this paper, the number of cross cell reconfiguration is less, which may help to reduce the production waiting time. However, compared with the modular virtual cell reconfiguration scheme, the inherited virtual cell reconfiguration scheme reduces the number of virtual cells and the maximum completion time, improves the utilization of equipment, reduces the logistics 
cost, and achieves better equipment sharing. By changing the combination of arrival orders and calculating and analyzing several cases, the inherited unit reconfiguration has improved the number of virtual units, machine sharing, equipment utilization and logistics cost.

Table3. Cmparation between rconstruction scheme and initial scheme

\begin{tabular}{ccc}
\hline & Reconstruction scheme & Initial scheme \\
\hline Cell-intra times & 49 & 36 \\
Logistics cost & 25256 & 25316 \\
utilization rate of equipment & $32.02 \%$ & $30.66 \%$ \\
Maximum completion time & 411.4 & 426 \\
\hline
\end{tabular}

\section{Conclusion}

This paper studies the dynamic construction of virtual manufacturing cell (VMC) under the random disturbance of machine failure. In the model construction, in addition to considering the factors in the existing model, the production cost based on time and space organization, the delay delivery cost and the production load between cells are also considered. On this basis, a new method of dynamic construction of VMC is proposed. Because the model is a multi-objective nonlinear integer programming model with high complexity, a PSO-GA hybrid algorithm is designed to solve the model. In this algorithm, the crossover and mutation operations in genetic algorithm are imitated to speed up the update of particle speed and position, expand the neighborhood search scope and improve the quality of the solution. Finally, an example is given to verify the feasibility of the proposed model and the effectiveness of the algorithm. At the same time, experiments show that the proposed dynamic construction method of virtual manufacturing cell can effectively reduce the number of cross cell production, improve the utilization rate of equipment, reduce the cost of cell production logistics, realize the sharing of equipment, and maintain the relative balance of equipment workload between cells without increasing the total completion time.

\section{References}

1. Tambuskar D.P., Narkhede B.E., Mahapatra S.S. A novel algorithm for virtual cellular manufacturing considering real life production factors[J]. International Journal of Services \& Operations Management, 2015, 20(2):246-258.

2. Arkat J., Ghahve H. Scheduling of virtual manufacturing cells with outsourcing allowed[J]. International Journal of Computer Integrated Manufacturing, 2014, 27(12):1079-1089.

3. Feng Liang, Richard Y.K. Fung. Coordination mechanism in real-time scheduling of Virtual Cellular Manufacturing Systems[J]. Proceedings of the Institution of Mechanical Engineers, Part B: Journal of Engineering Manufacture, 2016, 230(3):830843.

4. Hamedi M, Esmaeilian G R, Ismail $\mathrm{N}$, et al. Capability-based virtual cellular manufacturing systems formation in dual-resource constrained settings using Tabu Search[J]. Computers \& Industrial Engineering, 2012, 62(4):953-971.

5. Kesen S.E., Toksari M.D., Zülal Güngr, et al. Analyzing the behaviors of virtual cells (VCs) and traditional manufacturing systems: Ant colony optimization (ACO)-based metamodels[J]. Computers \& Operations Research, 2009, 36(7):2275-2285. 
6. Nomden G., Zee DJVD. Virtual cellular manufacturing: Configuring routing flexibility[J]. International Journal of Production Economics, 2008, 112(1):439-451.

7. Paydar M M, Saidi-Mehrabad M . Revised multi-choice goal programming for integrated supply chain design and dynamic virtual cell formation with fuzzy parameters[J]. International Journal of Computer Integrated Manufacturing, 2015, 28(3):251-265.

8. Iraj Mahdavi,Amin Aalaei,Mohammad Mahdi Paydar,Maghsud Solimanpur. Multiobjective cell formation and production planning in dynamic virtual cellular manufacturing systems[J]. International Journal of Production Research,2011,49(21):465-477.

9. Rezazadeh H., Ghazanfari M., Sadjadi S.J., et al. Linear programming embedded particle swarm optimization for solving an extended model of dynamic virtual cellular manufacturing systems[J]. Journal of Applied Research and Technology, 2009, 7(1):83-108.

10. Jon Bokrantz, Anders Skoogh, Torbjörn Ylipää, et al. Handling of production disturba-nces in the manufacturing industry[J]. Journal of Manufacturing Technology Management, 2016, 27(8):1054-1075. 